\title{
Presence of endogenous prednisolone in human urine ${ }^{\text {is }}$
}

\author{
Marco Fidani ${ }^{\mathrm{a}, *}$, Maria C. Gamberini $^{\mathrm{b}}$, Giuseppe Pompa ${ }^{\mathrm{c}}$, Francesca Mungiguerra ${ }^{\mathrm{a}}$, Alessio Casati $^{\mathrm{c}}$, \\ Francesco Arioli ${ }^{\mathrm{c}}$ \\ ${ }^{a}$ U.N.I.R.E. Lab. S.r.l., Via Gramsci 70, 20019 Settimo Milanese, MI, Italy \\ ${ }^{\mathrm{b}}$ Department of Pharmaceutical Sciences, Institute of Pharmacy, University of Modena and Reggio Emilia, Via Campi 183, 41125 Modena, Italy \\ ${ }^{\mathrm{c}}$ Department of Health, Animal Science and Food Safety, University of Milan, Via Celoria 10, 20133 Milan, Italy
}

\section{A R T I C L E I N F O}

\section{Article history:}

Received 10 July 2012

Received in revised form 17 October 2012

Accepted 26 October 2012

Available online 23 November 2012

\section{Keywords:}

Human urine

Prednisolone

Cortisol

Endogenous

\begin{abstract}
A B S T R A C T
The possibility of an endogenous presence of the glucocorticoid prednisolone has already been demonstrated in bovine and horse urine, with the aim of clarifying its origin in this matrix, which is used by official agencies for the control of illicit treatments. From this point of view, the endogenous nature of prednisolone could be a major topic in doping control of both amateur and professional human athletes. A study was therefore made on 34 human volunteers (13 males and 21 females; aged 22-62) to detect the presence of prednisolone in their urine by HPLC-MS ${ }^{3}$. One of the volunteers underwent vernal allergy treatment with betamethasone for two subsequent years. An investigation was carried out with the aim of verifying if the suppression, and the circadian rhythm, of cortisol urinary levels could also apply to prednisolone. The results of the study show that prednisolone was present in the urine of all 34 volunteers, with a concentration very close to 100-times lower that of cortisol, with no dependence on gender. The same ratio (1/100) was observed in the prednisolone and cortisol levels detected during the $24 \mathrm{~h}$ together with the suppression of prednisolone by betamethasone treatment.

These data demonstrate the endogenous nature of low concentrations of prednisolone in human urine, and motivate further studies about the biosynthetic pathways of this corticosteroid and its relationship with stress in humans, as already described in cows.
\end{abstract}

(c) 2012 Elsevier Inc. All rights reserved.

\section{Introduction}

Prednisolone (11 $\beta, 17 \alpha, 21$-trihydroxy-pregna-1,4-diene-3,20dione) is a glucocorticosteroid with a similar structure to cortisol (11 $\beta, 17 \alpha, 21$-trihydroxy-pregn-4-ene-3,20-dione), differing from this hormone by only one double bond at the $\mathrm{C} 1-\mathrm{C} 2$ position on ring A (Fig. 1). Prednisolone was first synthesised in 1955, through microbiological oxidation of cortisol [1]. Together with its prodrug prednisone, prednisolone is used both in human and veterinary medicine because of its anti-inflammatory and immunosuppressive activities [2,3]. The illicit use of prednisolone by athletes is monitored by the World Anti-Doping Agency (WADA), using incompetition controls for all corticosteroids administered by systemic (e.g. oral), non-systemic and non-topical (e.g. intra-articular, inhalation) routes [4] are performed. The Fédération Equestre Internationale $\left(\mathrm{FEI}^{\mathrm{TM}}\right)$ considers prednisolone as a "controlled medication substance" [5], i.e. a substance that must not be "present in the horse's body during an event" [6]. In the field of animal husbandry, prednisolone use is permitted in the European Union, with

\footnotetext{
Work originated from U.N.I.R.E. Lab. S.r.l., Via Gramsci 70, 20019 Settimo Milanese (MI), Italy.

* Corresponding author. Tel.: +39 0233502133; fax: +39 0233514200

E-mail address: m.fidani@unirelab.it (M. Fidani).
}

the restriction to meet the maximum residue limits (MRLs) in edible tissues [7]; its presence in the urine of food-producing animals is not, however, provided.

Some studies show that a problem in finding of prednisolone in human, equine and bovine urine exists: this corticosteroid could in fact be the product of a microbiological dehydrogenation of cortisol after the collection of urine samples [8-11], and endogenous metabolic pathways could also be involved in bovine [12] and equine [13] animals. The analogies between the anabolic steroid boldenone (17 $\beta$-hydroxy-1,4-androstadiene-3-one) and the glucocorticosteroid prednisolone have to be underlined: both steroids have a $\mathrm{C} 1-$ $\mathrm{C} 2$ double bond; cortisol is released by the adrenal gland in response to stimulation by adrenocorticotropic hormone (ACTH); also androstenedione (4-androsten-3,17-dione; AED), the direct precursor of testosterone (17 $\beta$-hydroxy-4-androsten-3-one) can be released by adrenals in response to the same stimulation [14]; and AED differs from the precursor of boldenone, androstadienedione (1,4-androstadiene-3,17-dione; ADD), in the same way that cortisol differs from prednisolone, i.e. the dehydrogenation of the carbon atoms in position 1 and 2. In a study carried out using GC/C/IRMS (gas chromatography/combustion/isotope ratio mass spectrometry), Piper et al. [15] showed that the ${ }^{13} \mathrm{C} /{ }^{12} \mathrm{C}$ isotope ratios of boldenone and its main metabolite $5 \beta$-androst-1-en-17 $\beta$-ol-3-one were inconsistent with an exogenous origin in 11 out of 23 athlete urine samples 


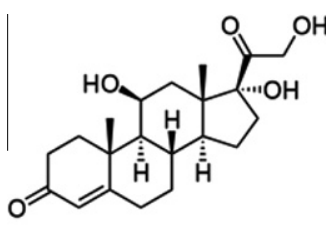

Cortisol

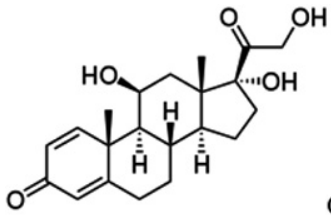

Prednisolone

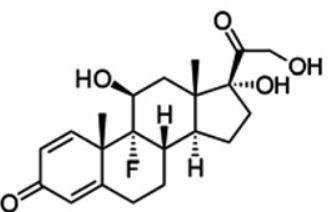

Betamethasone

Fig. 1. Chemical structures of cortisol, prednisolone and betamethasone.

found positive in a 4 -year period. As two of the 11 samples were collected from the same athlete within a one-month interval, the authors strongly affirmed the endogenous production of boldenone, likely in the gut, working as an "endocrine active side organ".

In the present work, it was hypothesised that prednisolone synthesis could be endogenous, similar to boldenone synthesis. The presence of the glucocorticosteroid in urine was therefore investigated in 34 healthy volunteers of both sexes in order to understand if the frequency of prednisolone detection and the correlation with cortisol urinary levels confirmed this supposition. The prednisolone urinary levels of a male volunteer, orally treated to prevent pollen allergy with betamethasone ( $9 \alpha$-fluoro-11 $\beta, 17 \alpha, 21$-trihydroxy-16 $\beta$-methylpregna-1,4-diene-3,20-dione) on medical prescription, were also investigated, in two different years, before, during and after the treatment.

\section{Experimental}

\subsection{Reagents and chemicals}

Cortisol, prednisolone, betamethasone and methylprednisolone (as internal standard) were from Sigma-Aldrich (St. Louis, MO, USA); tert-butyl methyl ether was from Panreac (Barcelona, Spain); acetonitrile, methanol and formic acid were from J.T. Baker (Deventer, Holland). All solvents used were of HPLC grade. Water was freshly prepared with a Milli-Q Advantage A10 Ultrapure Water Purification System (Merck-Millipore, Darmstadt, Germany). Standard stock solutions $(1 \mathrm{mg} / \mathrm{mL}$ ) were prepared by dissolving the dry powder of each analyte in methanol; solutions were stored at $-20^{\circ} \mathrm{C}$. Working solutions were prepared daily by diluting the stock solutions with methanol.

Bentelan $^{\circledR}$ (effervescent tablets 0.5 or $1 \mathrm{mg}$-betamethasone 21disodium phosphate 0.6578 or $1.316 \mathrm{mg}$ equal to betamethasone 0.5 or $1 \mathrm{mg}$, respectively) was from Defiante Farmacêutica, S.A., Funchal, Portugal.

\subsection{Study population}

In total, 34 healthy individuals (33 did not use glucocorticoids, one was studied for two subsequent years both before, during and after a treatment with a glucocorticoid) participated in this study. The urine samples from the volunteers (13 males aged 30-61; 21 females aged 22-62) were analysed. All individuals provided one urine sample, except for two male and three female individuals who gave two samples, collected within a six-month interval. One individual, a 41-year old male, gave urine samples nine times in a 24-h period before, and once a day (at 10.00 A.M.) during an oral treatment with betamethasone (a medical prescription to prevent pollen allergy) and 12 and $15 \mathrm{~h}$ after the last intake of the corticosteroid; in a second trial in the subsequent year, urine was collected from the same subject $1 \mathrm{~h}$ before the first administration, at the moment (11:00 P.M.) and 7 h (6:00 A.M.) after each oral administration of betamethasone and over 3.5 days after the last administration at different intervals by urinary urges. The dosages were: one $0.5-\mathrm{mg}$ tablet orally administered per day for 11 days at
10.00 A.M. (first trial) or one 1-mg tablet orally administered per day for 7 days at 11.00 P.M. (second trial).

All samples were analysed for the presence of prednisolone and cortisol; in the second trial concerning the betamethasone treatment, this corticosteroid was considered for analysis, too. All participants gave written informed consent.

\subsection{Sample collection}

All samples were collected in sterile urine containers. The ones from non-treated volunteers were immediately extracted and analysed, while the ones from the betamethasone-treated individual were placed in a freezer at $-18^{\circ} \mathrm{C}$, transferred to the laboratory, thawed and processed, avoiding any further storage.

\subsection{Sample preparation}

Urine samples $(5 \mathrm{~mL})$ were spiked with the internal standard methylprednisolone to give a final concentration of $10 \mathrm{ng} / \mathrm{mL}$. Tert-butyl methyl ether $(5 \mathrm{~mL})$ was added. After shaking in a vertical rotary shaker for $30 \mathrm{~min}$, the sample was centrifuged at $3000 \mathrm{~g}$ for $30 \mathrm{~min}$. The upper organic layer was collected with a Pasteur pipette, transferred to a $10-\mathrm{mL}$ glass tube and dried under vacuum in a centrifugal evaporator at a temperature of $45^{\circ} \mathrm{C}$. The residue was dissolved in $100 \mu \mathrm{L}$ of the mobile phase (30\% acetonitrile, $70 \%$ formic acid in a $0.1 \%$ aqueous solution) and transferred to vials for HPLC-MS 3 full-scan analysis. The injection volume was $5 \mu \mathrm{L}$.

\subsection{Sample analysis}

The HPLC-MS ${ }^{3}$ analysis (and the problems involved) was described in detail in a previous work [13]. Briefly, an LTQ linear ion trap mass spectrometer equipped with an ESI source (Thermo Fisher Scientific, San José, CA, USA), connected to a Surveyor Autosampler and an MS Pump (Thermo Fisher Scientific, San José, CA, USA), was used. The chromatographic separation was performed at ambient temperature, in the isocratic condition, on a reversedphase Sunfire ${ }^{\circledR}$ column $(150 \times 2.1 \mathrm{~mm}, 3.5 \mu \mathrm{m}$; Waters, Milford, MA, USA) equipped with a Sunfire $C_{18}$ Guard Column ${ }^{\circledR}$ $(2.1 \times 10 \mathrm{~mm}$ i.d., $3.5 \mu \mathrm{m})$. The mobile phase consisted of a mixture of $70 \%$ water with $0.1 \%$ formic acid and $30 \%$ acetonitrile at a flow rate of $0.3 \mathrm{~mL} / \mathrm{min}$. Methylprednisolone $(10 \mathrm{ng} / \mathrm{mL})$ was used as the internal standard. The linear ion trap mass spectrometer was operated in negative electrospray ionisation mode (ESI-) under the following conditions: sheath and auxiliary gas (nitrogen) flow rates of 40 and 20 arbitrary units, respectively; spray voltage of $4 \mathrm{kV}$; ion transfer capillary temperature of $275^{\circ} \mathrm{C}$; capillary voltage of $-6 \mathrm{~V}$; tube lens offset of $-50 \mathrm{~V}$. Helium was used for collision-induced dissociation.

Prednisolone showed, in full scan MS, the very abundant and stable formate adduct $405\left(\left[\mathrm{M}+\mathrm{HCOO}^{-}\right)\right.$. Consequently, this ion was used as precursor ion for the $\mathrm{MS}^{2}$ analysis. The most abundant ion detected after the collision had $m / z=329$, which was then used as a precursor for $\mathrm{MS}^{3}$ fragmentation. The analysis was performed in full-scan mode (Table 1). The ESI-MS parameters had been 
Table 1

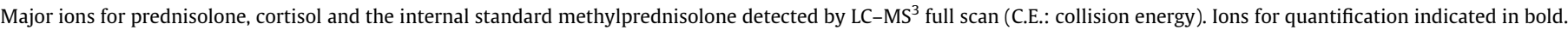

\begin{tabular}{|c|c|c|c|c|c|c|c|c|}
\hline \multirow[t]{2}{*}{ Compound } & \multicolumn{3}{|l|}{$\mathrm{MS}^{2}$} & \multicolumn{5}{|l|}{$\mathrm{MS}^{3}$} \\
\hline & \multirow{2}{*}{$\begin{array}{l}\left([\mathrm{M}+\mathrm{HCOO}]^{-}\right) \text {precursor ion }(\mathrm{m} / \mathrm{z}) \\
405\end{array}$} & \multirow{2}{*}{$\begin{array}{l}\text { C.E. (\%) } \\
15\end{array}$} & \multirow{2}{*}{$\begin{array}{l}\text { Precursor ion }(\mathrm{m} / \mathrm{z}) \\
329\end{array}$} & \multirow{2}{*}{$\begin{array}{l}\text { C.E. (\%) } \\
18.5\end{array}$} & \multicolumn{4}{|c|}{ Product ions $(\mathrm{m} / \mathrm{z})$} \\
\hline Prednisolone & & & & & 313 & 295 & 280 & 187 \\
\hline Cortisol & 407 & 20 & 331 & 23 & 297 & & & \\
\hline Methylprednisolone & 419 & 20 & 343 & 20 & 309 & & & \\
\hline
\end{tabular}

previously optimised by direct injection of a solution of prednisolone $(1 \mu \mathrm{g} / \mathrm{mL})$ at a flow rate of $20 \mu \mathrm{L} / \mathrm{min}$. Data acquisition and analysis were accomplished using Xcalibur ${ }^{\circledR}$ software version 1.4 (Thermo Fisher Scientific).

\subsection{Method validation}

Blank urine belonged to the subjects participating in the study. Betamethasone-free urine was easily found. As regards prednisolone and cortisol, blank urine came from the individual treated with betamethasone, from seventh day to the end of treatment. The absence of the studied corticosteroids was checked by the analytical method described above.

A calibration curve was thus prepared with blank urine and water samples, spiked to give known concentrations of betamethasone or prednisolone and cortisol, $(10,50,100,500 \mathrm{pg} / \mathrm{mL}, 1,10,100$ and $200 \mathrm{ng} / \mathrm{mL}$ ). Three replicates were measured on three different days after liquid-liquid extraction. The following parameters were calculated: precision, expressed as intra- and inter-day coefficient of variations (CV\%), on four blank urine samples, spiked with $100 \mathrm{pg} / \mathrm{mL}$, corresponding to twice the limit of quantification (LOQ); recovery (\%), based on three samples spiked at six different concentrations $(10,50,100,500 \mathrm{pg} / \mathrm{mL}, 1$ and $10 \mathrm{ng} / \mathrm{mL})$ in water and urine, calculated as (Observed value-Baseline value)/spike amount; limit of detection (LOD), defined as the lowest level at which a compound could be identified (signal-to-noise ratio greater than 3) and LOQ, defined as the lowest level at which a compound could be identified and quantified (signal-to-noise ratio greater than 10); between-run accuracy, on three different days using four different samples spiked with $100 \mathrm{pg} / \mathrm{mL}$ (twice the LOQ).

\subsection{Statistical analysis}

The Kolmogorov and Smirnov method was used to test the assumption of a Gaussian distribution. Unpaired $t$-test was used when the assumption was confirmed to compare males and females, otherwise a non-parametric Mann-Whitney test was used. The correlation between cortisol and prednisolone urinary concentrations was checked through the non-parametric, Spearman Rank Correlation test except in the 24-h period before treatment of the first trial on the treated individual where the Pearson correlation was used, due to the Gaussian distribution of the values. Because of the similarity of the results, no statistical tests were performed in the second trial. The software used was GraphPad InStat ${ }^{\mathrm{TM}}$ version 3.00 (GraphPad Software, San Diego, California USA; www.graphpad.com).

\section{Results}

\subsection{Identification of prednisolone}

In a previous study in our laboratories on prednisolone presence in equine urine, [13] we referred to the difficulties found by some Authors in detecting prednisolone. The analysis of urine samples was therefore carried out following the experience made on that matrix. After preliminary trials (data not shown) that confirmed the conclusions already drawn, the full-scan $\mathrm{LC} \mathrm{MS}^{3}$ with a linear ion trap was so chosen.

\subsection{Method validation}

The correlation coefficient was 0.99 for each corticosteroid. The intra-day CVs\% ranged between 6.8 and 8.2, the inter-day CVs\% ranged between 7.8 and 11.2 for all corticosteroids. The recoveries ranged from $89 \%$ to $94 \%$ in urine and water, indicating the absence of a matrix effect. The LOD was $10 \mathrm{pg} / \mathrm{mL}$ and the LOQ was $50 \mathrm{pg} /$ $\mathrm{mL}$ for each corticosteroid. The between-run accuracy was $4.2 \%$ for prednisolone, $2.8 \%$ for cortisol and $3.4 \%$ for betamethasone.

\subsection{Quantification and statistical analysis}

Prednisolone and cortisol were detected in urine samples from all 34 individuals. The concentrations, expressed as mean \pm SD, of cortisol were $55.13 \pm 35.06$ and $37.13 \pm 39.61 \mathrm{ng} / \mathrm{mL}$, and of prednisolone were $440 \pm 360$ and $470 \pm 580 \mathrm{pg} / \mathrm{mL}$, in male and female urine respectively. The Kolmogorov and Smirnov method showed normal distributions for cortisol and prednisolone in both male and female urine. The unpaired $t$-test, made to compare the urinary levels between genders, did not show significant differences for cortisol (two-tailed $P$-value $=0.1885$ ) and prednisolone (two-tailed $P$ value $=0.8450$ ). Due to this fact, the concentrations of cortisol and prednisolone in male and female urine were merged. The means and SD from all the donors were recalculated and the final urinary concentration values were $44.01 \pm 38.42 \mathrm{ng} / \mathrm{mL}$ for cortisol and $460 \pm 500 \mathrm{pg} / \mathrm{mL}$ for prednisolone. The concentration ratios of prednisolone/cortisol, calculated for all samples, passed the normality test and the mean value was $0.01 \pm 0.005$ with the $95 \%$ confidence limits 0.008 and 0.011 . The normality test was made again on merged data and only cortisol resulted in a normal distribution. Finally the correlation between cortisol and prednisolone was checked through the Spearman's test. A very good positive correlation was found: the Spearman $r$ value was 0.8527 with an extremely significant two-tailed $P<0.0001$. The non-parametric Spearman correlation was used because prednisolone did not follow a Gaussian assumption and corresponds to the Pearson correlation for populations that follow the Gaussian distribution: in general a Pearson $r$ from 0.7 to 1 is considered to be a strong association [11].

Urine samples of five out of 34 individuals were collected and analysed again within a six-month interval and prednisolone was always detected. The mean \pm SD concentrations of urinary prednisolone from these five volunteers only were: $910 \pm 390 \mathrm{pg} / \mathrm{mL}$ for the first collection and $650 \pm 830 \mathrm{pg} / \mathrm{mL}$ for the second collection. Cortisol was also detected and the corresponding values were $90.7 \pm 51.6$ and $58.6 \pm 58.4 \mathrm{ng} / \mathrm{mL}$. A very good positive Spearman correlation between prednisolone and cortisol levels in both sets of samples was found as the values were 0.83 and 0.98 , respectively.

\subsection{Trials on an individual treated with betamethasone}

\subsubsection{First trial}

The urine of the 41-year old male volunteer showed a correlation between prednisolone and cortisol both in the 24-h period 


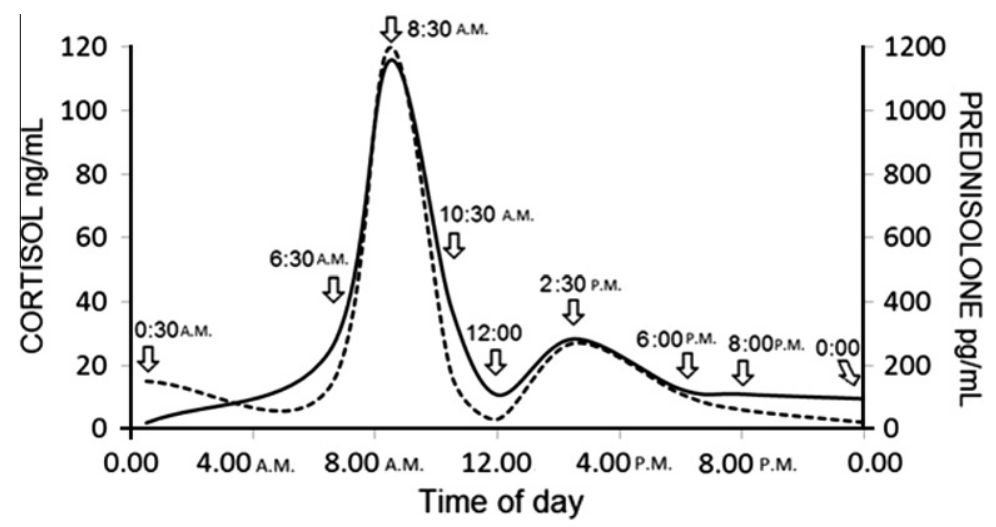

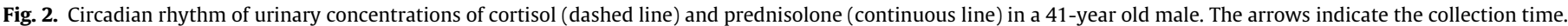

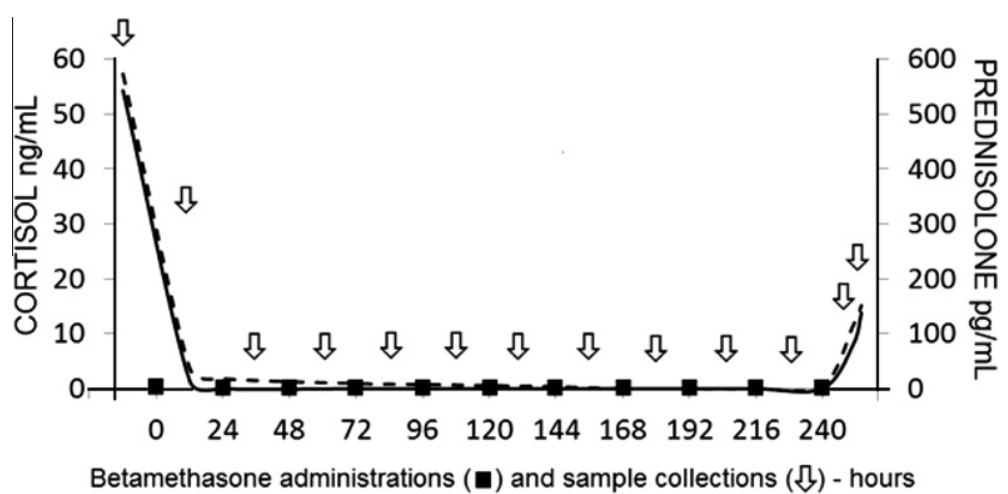

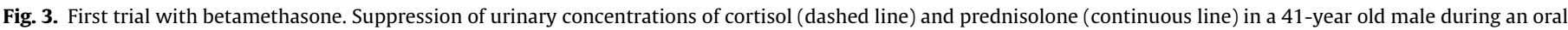

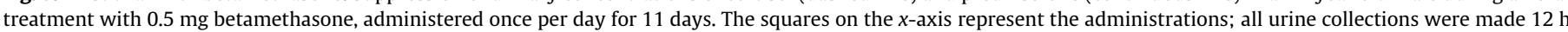
after each administration and 12 and $15 \mathrm{~h}$ after the last one.

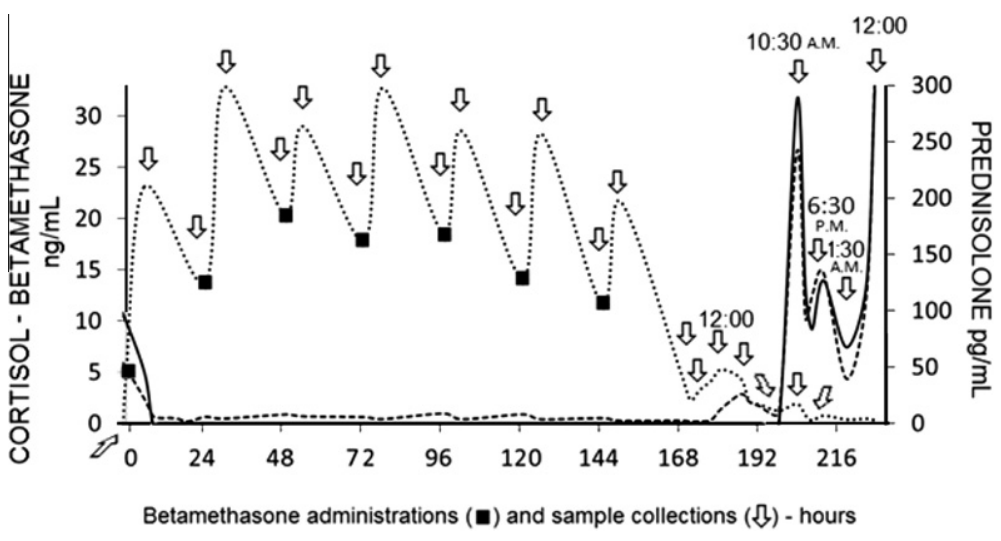

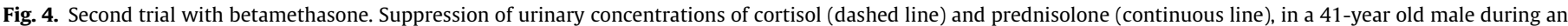

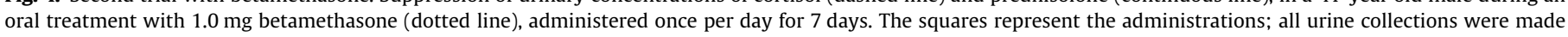

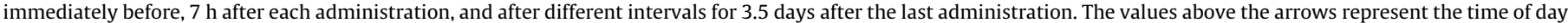
in which the urine collections were made.

before the treatment and in the period of the oral treatment. The Pearson $r$ in the period before the treatment was 0.97, and the Spearman $r$ during the treatment was 0.75 . In Fig. 2 the daily variation of cortisol and prednisolone urinary concentrations, similar to the circadian rhythm of plasma cortisol [16], is shown with a peak at 8.30 A.M. for both cortisol $(116 \mathrm{ng} / \mathrm{mL})$ and prednisolone $(1.238 \mathrm{ng} / \mathrm{mL})$. In Fig. 3, the suppression of cortisol and prednisolone is shown following the betamethasone administration. The urinary concentrations of prednisolone and cortisol, measured on samples collected at $10.00 \mathrm{AM}$, dramatically decreased after the first treatment, from 543 to $0 \mathrm{pg} / \mathrm{ml}$ and from 57.3 to $3.83 \mathrm{ng} / \mathrm{mL}$, respectively. Prednisolone was not detected during the treatment, while cortisol slowly decreased to $0 \mathrm{ng} / \mathrm{ml}$ at day seven. In the urine sample collected $15 \mathrm{~h}$ after the last betamethasone administration, prednisolone and cortisol were detected again at the concentration of $138 \mathrm{pg} / \mathrm{mL}$ and $15.1 \mathrm{ng} / \mathrm{mL}$, respectively. 


\subsubsection{Second trial}

The same subject underwent a betamethasone treatment in the subsequent year. The urinary concentrations of cortisol and prednisolone at the moment of the first tablet administration (11.00 P.M.) were $5.92 \mathrm{ng} / \mathrm{mL}$ and $97.9 \mathrm{pg} / \mathrm{mL}$, respectively. Betamethasone concentrations showed a fluctuation from 11.6 to $32.8 \mathrm{ng} / \mathrm{mL}$ during the treatment, and its minimum levels corresponded to the sample collections made at the moment of the oral administration. The fluctuation was observed for cortisol also, but the concentrations were inversely proportional: when betamethasone raised, cortisol decreased. However, cortisol concentrations were very low and ranged between 170 and 770 pg/mL. Prednisolone was not found eight hours after the first betamethasone intake and was detected again 2.5 days after the last treatment $(287 \mathrm{pg} / \mathrm{mL})$, together with a peak of cortisol at 10.30 A.M $(26.6 \mathrm{ng} / \mathrm{mL})$, whose concentration was raised from the 37 th hour after the last administration. The circadian rhythm of cortisol and prednisolone, checked after betamethasone administration, were also observed in this second trial. In Fig. 4, the urinary concentrations of betamethasone, cortisol and prednisolone detected in this trial are shown.

\section{Discussion}

The LC-MS ${ }^{3}$ method used in this work considered, for identification and quantification of prednisolone, the transitions $329 \rightarrow 280$ and $329 \rightarrow 187$. The reason for this choice is analogous to those already exposed regarding horse urine [13]. A peak with the same retention time of prednisolone, but a nonoverlapping spectrum was present in the chromatogram. The $m / z 405$, the parent ion represented by the formate adduct of prednisolone $\left(\left[\mathrm{M}+\mathrm{HCOO}^{-}\right)\right.$, dissociated at $359 \mathrm{~m} / z$ (i.e. molar weight of prednisolone minus 1) and $329 \mathrm{~m} / \mathrm{z}$ (i.e. molar weight of prednisolone minus a $\mathrm{CH}_{2} \mathrm{OH}$ group). The $\mathrm{m} / \mathrm{z} 359$ originated from the matrix, while the $m / z 329$ was very abundant and proved to be an excellent parent ion for the $\mathrm{MS}^{3}$ dissociation. The transitions $329 \rightarrow 313$ and $329 \rightarrow 295$ gave rise to possible misinterpretations, as they were present in another peak, close to the prednisolone retention time (Fig. 5A and B1). The two $\mathrm{m} / \mathrm{z} 280$ and 187, which were not present in other peaks (Fig. 5B2), proved to be very characteristic ions in the $\mathrm{MS}^{3}$ method, and were therefore used for identification and quantification.

All urine samples showed the presence of both prednisolone and cortisol and their concentration ratio was about 0.01 , with a narrow range for the $95 \%$ confidence limits. This consistency is the first evidence of the endogenous nature of prednisolone, considered as a by-product or a metabolite of cortisol. Secondly, it is interesting to note that the same proportion of $\sim 1 / 100$ between prednisolone and cortisol was observed in the collections of the 24-h study as is shown in Figs. 2 and 4. Thirdly, the inhibitory effect of betamethasone concerns both cortisol and prednisolone, even if in this last case the $1 / 100$ ratio is not observable because of the absence of prednisolone in the urine collected from 24 to $240 \mathrm{~h}$ and from 8 to $203.5 \mathrm{~h}$ in first and second trials, respectively, and of cortisol from 168 to $216 \mathrm{~h}$ in the first trial. These observations reinforce each other, the last two confirming the first one - i.e. prednisolone can have an endogenous origin in man - as already observed in cow and horse [12,13]. The possibility of an ex vivo conversion of cortisol to prednisolone [8] was in advance considered. We therefore eliminated the sample storage (untreated volunteers) or minimized it (treated individual). Preliminary trials, performed by placing the urine samples in the freezer at $-18^{\circ} \mathrm{C}$ for one day, showed no changes in cortisol and prednisolone levels (personal data, not shown). A
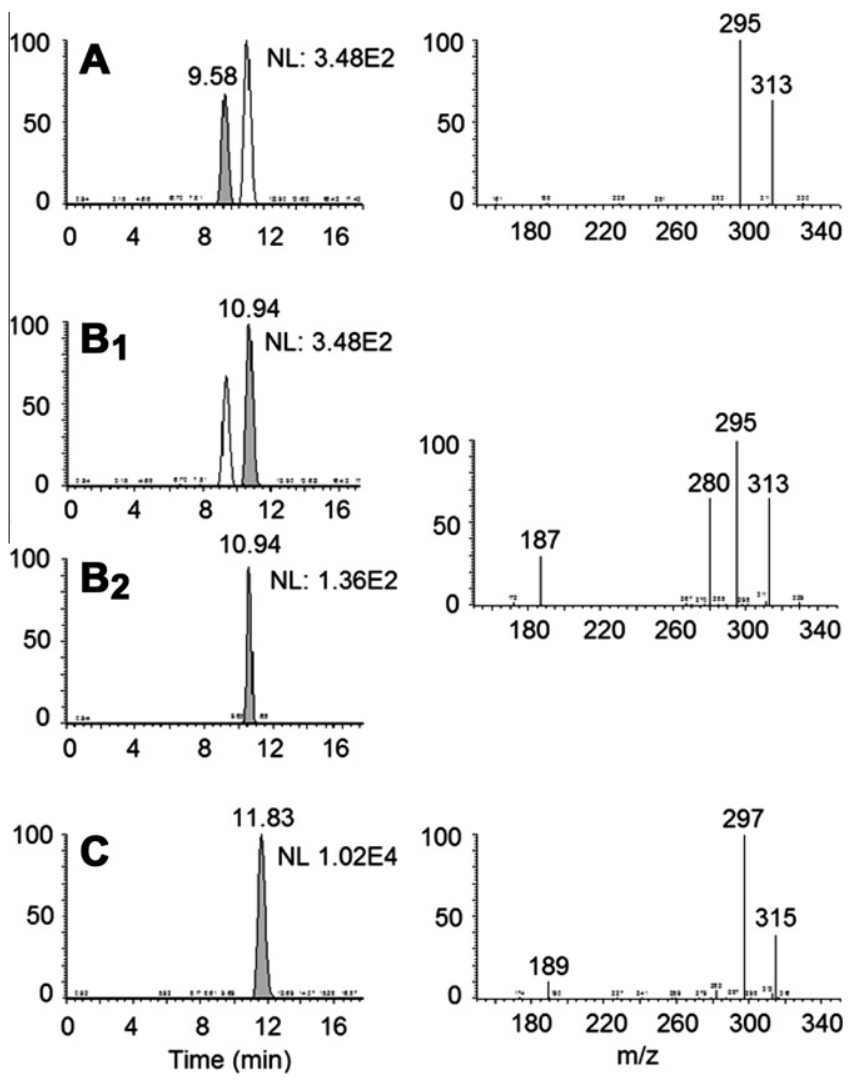

Fig. 5. Representative full mass LC-MS3 extracted chromatogram depicting the analysis of a human urine sample. The spectra show the total ion current at the Rt of the interfering peak (A), prednisolone (B1 and B2) and cortisol (C). In B1 the four transitions $329 \rightarrow 313 ; 329 \rightarrow 295 ; 329 \rightarrow 280$ and $329 \rightarrow 187$ are considered; in B2 only the $m / z 280$ and 187 .

criticism could be made about the fact that the study on the circadian rhythm linked to the betamethasone-induced suppression was made on only one volunteer. It has to be noted that this work was made as a "natural" continuation of the studies made on bovine and horse and that the availability of a betamethasone-treated subject was not provided, but that coincidentally one of the volunteers had to undergo anti-allergic vernal treatments. Because of our inability to plan the betamethasone treatment, the collection protocols in the two trials are also different. It seems likely, however, that cortisol and prednisolone take more than two days to return to the pre-treatment urinary concentrations: in the second trial, the circadian rhythm of the corticosteroids is again evident only at the third day after the treatment, even if levels are lower than in the first trial. It cannot be excluded, however, that other factors - e.g. different physiological or stress conditions - could affect the concentration values as well as the delays in the maximum corticosteroid levels.

This study is the first related to endogenous prednisolone in humans and, even if further investigations aimed at the demonstration of the endogenous origin of this corticosteroid could be not necessary, the deepening of the knowledge about the relationship that seems to exist between prednisolone and cortisol could be a reason to continue this work. In particular, the confirmation of the concentration ratio in pathological and stress conditions, the study of the metabolic pathways leading to the prednisolone synthesis could represent major topics for the study of a possible role of this corticosteroid. 


\section{References}

[1] Nobile A, Charney W, Perlman PL, Herzog HL, Payne CC, Tully ME, Jevnik MA Hershberg EB. Microbiological transformation of steroids I. $\Delta 1,4$-Diene-3ketosteroids. Am J Chem Soc 1955;77:4184.

[2] Porter RS, Kaplan JL. The Merck manual online. Whitehouse Station, (NJ) USA: Merck Sharp \& Dohme Corp. <http://www.merckmanuals.com/professional/ index.html $>$ [retrieved 27.06.12].

[3] Kahn CM, Line S, Aiello SE. The Merck veterinary manual, online. 9th ed. Whitehouse Station, (NJ) USA: Merck Sharp \& Dohme Corp.; 2005. <http:// www.merckvetmanual.com/mvm/index.jsp?cfile=htm/bi/mvm_bkindex.htm> [retrieved 27.06.12].

[4] World Anti-doping Agency. The 2011 prohibited list international standard international standard, 18 September 2010.

[5] <http://prohibitedsubstancesdatabase.feicleansport.org/ list.php?q=prednisolone $>$ [retrieved 27.06.12]

[6] FEI Equine Anti-Doping and Controlled Medication Regulations. 1st ed. Effective 5 April 2010.

[7] EEC Commission Regulation No. 37/2010. Off J Eur Community L 15:1-72.

[8] Bredehöft M, Baginski R, Parr MK, Thevis M, Schänzer W. Investigations of the microbial transformation of cortisol to prednisolone in urine samples. J Steroid Biochem Mol Biol 2012;129:54-60.

[9] Arioli F, Fidani M, Casati A, Fracchiolla ML, Pompa G. Investigation on possible transformations of cortisol, cortisone and cortisol glucuronide in bovine faecal matter using liquid chromatography-mass spectrometry. Steroids 2010;75:350-4.
[10] Arioli F, Casati A, Fidani M, Silvestri M, Pompa G. Prednisolone and prednisone neo-formation in bovine urine after sampling. Animal 2012;6:1023-9.

[11] Ferranti G, Delli Quadri F, Palleschi L, Ferretti G, Marchiafava C, Pezzolato M Bozzetta E, Caramelli M, Draisci R. Studies on the presence of natural and synthetic corticosteroids in bovine urine. Steroids 2011;76:616-25.

[12] Pompa G, Arioli F, Casati A, Fidani M, Bertocchi L, Dusi G. Investigation of the origin of prednisolone in cow urine. Steroids 2011;76:104-10.

[13] Fidani M, Pompa G, Mungiguerra F, Casati A, Fracchiolla ML, Arioli F. Investigation of the presence of endogenous prednisolone in equine urine by high-performance liquid chromatography mass spectrometry and highresolution mass spectrometry. Rapid Commun Mass Spectrom 2012;26:879-86.

[14] Schimmer BP, Funder JW. In: Brunton LL, Chabner BA, Knollmann C Blumenthal D, Murri N, Hilal-DAndan R, editors, Goodman \& Gilman's the pharmacological basis of therapeutics. ACTH, adrenal steroids, and pharmacology of the adrenal cortex. Section V. Hormones and hormone antagonists, 12th online ed. New York, USA: The Mcgraw-Hill Company. <http://www.accessmedicine.com/resourceTOC.aspx?resourceID=651> [Chapter 42, retrieved 27.06.12].

[15] Piper T, Geyer H, Gougoulidis V, Flenker U, Schänzer W. Determination of ${ }^{13} \mathrm{C} /{ }^{12} \mathrm{C}$ ratios of urinary excreted boldenone and its main metabolite $5 \beta$ androst-1-en-17b-ol-3-one. Drug Test Anal 2010;2:217-24.

[16] Rohatagi S, Bye A, Mackie AE, Derendorf H. Mathematical modelling of cortisol circadian rhythm and cortisol suppression. Eur J Pharm Sci 1996;4:341-50. 